\title{
Association between urinary 8-OHdG and pulse wave velocity in hypertensive patients with type 2 diabetes mellitus
}

Kazuhiko Kotani ${ }^{1}$, PhD, MD, Toshiyuki Yamada ${ }^{1}$, PhD, MD

\begin{abstract}
INTRODUCTION Oxidative stress, assessed using 8-hydroxy-2'-deoxyguanosine (8-OHdG), can be associated with arterial stiffness in patients with type 2 diabetes mellitus (T2DM) and/or hypertension (HT). We investigated the correlation between urinary 8-OHdG and pulse wave velocity (PWV) in hypertensive and non-hypertensive T2DM patients with fair glycaemic control to determine the clinical significance of HT as a comorbidity in the diabetic state. METHODS Clinical data, including traditional cardiovascular risk factors, diabetic complications, prescribed agents, urinary 8-OHdG level and brachial-ankle PWV, was collected from T2DM patients with and without HT.

RESULTS There were 76 patients (45 men, 31 women; mean age 61 years; mean haemoglobin A1c level 6.5\%) in the study cohort. T2DM patients with HT had significantly higher mean PWV than patients without HT $(1,597 \mathrm{~cm} / \mathrm{s}$ vs $1,442 \mathrm{~cm} / \mathrm{s} ; \mathrm{p}<0.05)$. Patients with HT showed no significant difference in 8 -OHdG levels relative to those without HT (median $7.9 \mathrm{ng} / \mathrm{mg}$ creatinine vs $8.8 \mathrm{ng} / \mathrm{mg}$ creatinine; $\mathrm{p}>0.05$ ). Simple linear correlation and stepwise multiple linear regression analyses revealed that 8-OHdG levels correlated independently, significantly and positively with PWV among T2DM patients with HT $(r=0.33, p<0.05 ; \beta=0.23, p<0.05)$. No significant correlation was observed between 8-OHdG levels and PWV among T2DM patients without HT.

CONCLUSION In the hypertensive state, oxidative stress can be responsible for the development of arterial stiffness, even in patients with fairly well controlled T2DM. Oxidative stress management may be necessary for the prevention of cardiovascular disease in this population.
\end{abstract}

Keywords: arterial stiffness, atherosclerosis, DNA damage, oxidative stress, pulse wave velocity

\section{INTRODUCTION}

The Asia-Pacific region is facing an epidemic of type 2 diabetes mellitus (T2DM), paralleled by high rates of cardiovascular disease (CVD). ${ }^{(1,2)}$ Hypertension (HT) remains a major risk factor for CVD in the region. ${ }^{(3)}$ Further compounding matters, studies report that hyperglycaemia, increased blood pressure and oxidative stress can individually and/or concomitantly cause vascular damage, such as increased arterial stiffness. ${ }^{(4-6)}$ The degree of arterial stiffness is often estimated using modalities such as pulse wave velocity (PWV); in fact, PWV is currently being used as a surrogate index for future CVD outcome. ${ }^{(5)}$

On DNA oxidation, a hydroxyl group is added to the guanine molecule to form 8-hydroxyguanine, which on oxidative modification yields the product, 8-hydroxy-2'deoxyguanosine (8-OHdG)..$^{(7,8)}$ Hyperglycaemia promotes glucose oxidation and protein glycation, impairs DNA repair with resultant DNA cleavage, and generates reactive oxygen species, thereby leading to increased oxidative stress. ${ }^{(4,7,8)}$ Similarly, HT is accompanied by the formation of advanced glycation end products, and thereby increased oxidative stress and DNA damage. ${ }^{(9,10)}$ For these reasons, the measurement of 8 -OHdG has been used by various researchers to evaluate the DNA oxidation associated with the pathophysiology of T2DM and HT. ${ }^{(4,11-16)}$

A study by Nishikawa et al found that urinary $8-\mathrm{OHdG}$ levels were increased in T2DM patients with poor glycaemic control, (4) while a study by Espinosa et al reported that urinary $8-\mathrm{OHdG}$ levels were increased in patients with $\mathrm{HT}^{\left({ }^{(13)}\right.}$ However, Roselló-Lletí et al demonstrated that patients with HT and DM do not exhibit any differences with regard to $8-\mathrm{OHdG}$ levels when compared to patients with HT but no DM. ${ }^{(14)}$ Espinosa et al also reported that 8 -OHdG levels were reduced following antihypertensive treatment in patients with HT. ${ }^{(13)}$ Studies suggest lowered urinary $8-\mathrm{OHdG}$ levels upon intervention with antidiabetic agents in T2DM patients, ${ }^{(15)}$ or antihypertensive and antidiabetic agents in patients with T2DM and HT. ${ }^{(16)}$ Such changes in $8-\mathrm{OHdG}$ levels are positively correlated with readings taken on the cardio-ankle vascular index, which is also an arterial stiffness index (although it is different from PWV both in principle and clinical feature). ${ }^{(15,16)}$

While the occurrence of arterial stiffness and CVD have been reported even in non-severe hyperglycaemic states, ${ }^{(17)}$ Barengo and Tuomilehto reported that the presence of HT as a comorbidity in the hyperglycaemic state increased CVD

${ }^{1}$ Department of Clinical Laboratory Medicine, Jichi Medical University, Tochigi, Japan

Correspondence: Dr Kazuhiko Kotani, Research Head, Department of Clinical Laboratory Medicine, Jichi Medical University, $3311-1$ Yakushiji, Shimotsuke-City, Tochigi, 329-0498, Japan. kazukotani@jichi.ac.jp 
risk in patients with DM. ${ }^{(18)}$ These findings suggest that, aside from glycaemic control, HT and oxidative stress could also contribute to the development of T2DM-related CVD. Although the study by Roselló-Lletí et al found that the coexistence of HT and DM did not always enhance urinary 8-OHdG levels, the arterial stiffness of the patients was not evaluated by these authors. ${ }^{(14)}$ Masugata et al, on the other hand, found a positive correlation between urinary 8-OHdG and the cardio-ankle vascular index in patients with $\mathrm{HT}^{(19)}$ However, this study did not compare findings in patients with HT against patients without HT or patients with DM.

To the best of our knowledge, the association between urinary 8-OHdG and PWV in T2DM patients with fair glycaemic control in the presence or absence of comorbid HT has not yet been fully documented in the literature. The present study was thus designed to address this lacuna and determine whether a relationship exists between $8-\mathrm{OHdG}$ levels and brachial-ankle PWV readings (which is an often-used modality in the clinical setting ${ }^{(20)}$ ) in patients with fairly well controlled T2DM and comorbid HT.

\section{METHODS}

The study cohort included 76 patients with T2DM (45 men, 31 women; age range 35-81 years). Patients who had achieved fair glycaemic control (based on haemoglobin A1c [HbA1c] levels of $8.0 \%$, as previously described by Oomichi et $\left.\mathrm{a}^{(21)}\right)$ with diet and/or antidiabetic agent therapy were included. Patients with a history of cardiovascular or cerebrovascular disorders, thyroid disorders, haematological disturbances, and collagen, severe hepatic or renal disease were excluded. The study was approved by the ethics committee of Jichi Medical University, Tochigi, Japan. Informed consent was obtained from all patients enrolled in the study.

Current smoking habits were ascertained via interviews. Information on prescribed agents (e.g. antidiabetic, antihypertensive and/or lipid-lowering agents) was obtained from the medical records. Clinical measurements were obtained after overnight fasting. Body mass index (BMI) was calculated based on the weight and height measurements taken when patients were barefoot and wearing light clothing. Blood pressure (BP) was determined in the patient's right arm using a mercury sphygmomanometer with the patient in a seated position. Patients with systolic BP $\geq 140 \mathrm{mmHg}$ and/or diastolic BP $\geq$ $90 \mathrm{mmHg}$ and/or those consuming antihypertensive agents were deemed to have $\mathrm{HT}^{(22)}$

Mean BP (MBP) was calculated using the following equation: $\mathrm{MBP}=$ diastolic $\mathrm{BP}+($ systolic $\mathrm{BP}-$ diastolic $\mathrm{BP}) / 3$. Serum total cholesterol, high-density lipoprotein-cholesterol (HDL-C), triglycerides, creatinine and plasma glucose concentrations were enzymatically measured. Estimated glomerular filtration rate (eGFR) was calculated using the Modification of Diet in Renal Disease Study Group formulae; ${ }^{(23)}$ $\mathrm{eGFR}(\mathrm{men})=0.741 \times 175 \times$ age $^{-0.203} \times$ serum creatinine $\mathrm{e}^{-1.154}$ and eGFR (women) $=0.741 \times 175 \times$ age $^{-0.203} \times$ serum creatinine $^{-1.154} \times 0.742$. HbA1c levels were determined using high-performance liquid chromatography. Urinary 8-OHdG level was measured using enzyme-linked immunosorbent assays (Japan Institute for the Control of Aging, Shizuoka, Japan), with interassay and intra-assay coefficients of variation fixed at $2.1 \%$ and $4.5 \%$, respectively. ${ }^{(24,25)}$ Urinary samples were collected as spot urine from each patient in the morning before breakfast (7:30-10:00 am; around the same time as blood collection). The collected urinary samples were centrifuged at $800 \mathrm{~g}$ for 10 mins and the supernatant was used for assaying. 8 -OHdG measurements were expressed in terms of its ratio to creatinine in the same sample (i.e. /mg creatinine). Urinary albumin was measured using the turbidimetric immunoassay method (Wako Pure Chemical Industries Ltd, Osaka, Japan). Diabetic nephropathy was defined in terms of the ratio of albumin to creatinine in the same sample (i.e./mg creatinine), as albumin/creatinine ratio $(\mathrm{ACR}) \geq 30 \mu \mathrm{g} / \mathrm{mg}$ creatinine. ${ }^{(26)}$

Brachial-ankle PWV measurements were taken using a volume-plethysmographic apparatus (Form/ABI, Colin Medical Technology Co Ltd, Komaki, Japan), with interobserver and intraobserver coefficients of variation fixed at $8.4 \%$ and $10.0 \%$, respectively. ${ }^{(20)}$ The cuffs were wrapped around both brachia and ankles, with patients resting in the supine position. Measurements were obtained in a single (one-time) reading following a 5 -min rest. The pulse volume waveforms at the brachium and ankle were recorded using a semiconductor pressure sensor. Additionally, the presence of diabetic retinopathy - defined as nonproliferative and/or proliferative retinopathy - was determined by ophthalmoscopic examination. ${ }^{(27)}$

Data was expressed as mean \pm standard deviation or median (interquartile range). Differences in data between groups were examined using the unpaired $t$-test or chi-square test. A simple linear correlation test (Pearson's test) and stepwise multiple linear regression analysis were used to observe the correlation between PWV and other measured variables, including 8-OHdG. Stepwise multiple linear regression analysis was used to evaluate PWV with respect to the following variables: age, gender, current smoking habit, duration of DM, BMI, MBP, total cholesterol, HDL-C, triglycerides, HbA1c, creatinine, 8-OHdG, eGFR, nephropathy, retinopathy, and the use of antidiabetic, antihypertensive or lipid-lowering agents. Plasma glucose and ACR were not included as variables for multiple regression due to the colineality and biological nature of HbA1c and nephropathy, respectively. The duration of DM, triglycerides, 8-OHdG and ACR values were log-transformed for multiple linear regression analysis due to their skewed distributions. Statistical significance was defined at a p-value of $<0.05$.

\section{RESULTS}

Table I presents the demographic and clinical characteristics of the study population. T2DM patients with HT had a 
Table I. Demographic and clinical characteristics of patients with type 2 diabetes mellitus (T2DM).

\begin{tabular}{|c|c|c|c|}
\hline Variable & $\begin{array}{c}\text { T2DM patients } \\
\text { without HT }(n=31)\end{array}$ & $\begin{array}{l}\text { T2DM patients } \\
\text { with HT }(n=45)\end{array}$ & p-value* \\
\hline Age (yrs) & $61 \pm 10$ & $62 \pm 9$ & 0.56 \\
\hline $\begin{array}{l}\text { Gender } \\
\text { Men } \\
\text { Women }\end{array}$ & $\begin{array}{l}16(52) \\
15(48)\end{array}$ & $\begin{array}{l}23(51) \\
22(49)\end{array}$ & 0.91 \\
\hline Current smoking habit & $7(23)$ & $4(9)$ & 0.10 \\
\hline Duration of DM (yrs) & $3(0.5-7)$ & $7(2-15)$ & $<0.01^{*}$ \\
\hline BMI $\left(\mathrm{kg} / \mathrm{m}^{2}\right)$ & $24.1 \pm 3.7$ & $26.0 \pm 4.8$ & 0.07 \\
\hline MBP (mmHg) & $88 \pm 7$ & $101 \pm 10$ & $<0.01^{*}$ \\
\hline Total cholesterol (mmol/L) & $5.31 \pm 1.08$ & $5.04 \pm 0.77$ & 0.21 \\
\hline HDL-C (mmol/L) & $1.58 \pm 0.56$ & $1.52 \pm 0.45$ & 0.59 \\
\hline Triglycerides (mmol/L) & $1.15(0.94-1.46)$ & $1.38(0.99-1.95)$ & 0.26 \\
\hline Plasma glucose (mmol/L) & $6.37 \pm 1.48$ & $7.14 \pm 1.63$ & $0.04^{+}$ \\
\hline HbA1c (\%) & $6.2 \pm 1.0$ & $6.6 \pm 0.9$ & 0.06 \\
\hline Creatinine $(\mu \mathrm{mol} / \mathrm{L})$ & $63.0 \pm 14.1$ & $64.4 \pm 22.0$ & 0.76 \\
\hline 8-OHdG (ng/mg creatinine) & $8.8(6.9-10.5)$ & $7.9(6.2-10.0)$ & 0.36 \\
\hline PWV (cm/s) & $1,442 \pm 201$ & $1,597 \pm 307$ & $0.02^{+}$ \\
\hline eGFR $\left(\mathrm{mL} / \mathrm{min} / 1.73 \mathrm{~m}^{2}\right)$ & $79.3 \pm 19.8$ & $79.2 \pm 19.1$ & 0.99 \\
\hline ACR ( $\mu \mathrm{g} / \mathrm{mg}$ creatinine) & $11(8-21)$ & $20(11-66)$ & $0.04^{+}$ \\
\hline Nephropathy & $6(19)$ & $18(40)$ & 0.06 \\
\hline Retinopathy & $5(16)$ & $19(42)$ & $0.02^{+}$ \\
\hline $\begin{array}{l}\text { Antidiabetic agents } \\
\text { Sulfonylureas } \\
\text { Biguanides } \\
\text { Thiazolidinedione } \\
\alpha \text {-glucosidase inhibitors } \\
\text { Incretin-related agents }\end{array}$ & $\begin{aligned} 14 & (45) \\
9 & (29) \\
6 & (19) \\
2 & (6) \\
3 & (10) \\
4 & (13)\end{aligned}$ & $\begin{aligned} 31 & (69) \\
23 & (51) \\
12 & (27) \\
6 & (13) \\
7 & (16) \\
14 & (31)\end{aligned}$ & $\begin{array}{l}0.04^{+} \\
0.06 \\
0.46 \\
0.34 \\
0.46 \\
0.07\end{array}$ \\
\hline $\begin{array}{l}\text { Antihypertensive agents } \\
\text { ARBs or ACE inhibitors } \\
\text { Calcium channel blockers } \\
\beta \text {-blockers } \\
\text { Diuretics }\end{array}$ & $\begin{array}{l}0(0) \\
0(0) \\
0(0) \\
0(0) \\
0(0)\end{array}$ & $\begin{aligned} 34 & (76) \\
28 & (62) \\
24 & (53) \\
4 & (9) \\
6 & (13)\end{aligned}$ & $\begin{aligned}< & 0.01^{*} \\
< & 0.01^{*} \\
< & 0.01^{*} \\
& 0.09 \\
& 0.03^{+}\end{aligned}$ \\
\hline $\begin{array}{l}\text { Lipid-lowering agents } \\
\text { Statins } \\
\text { Fibrates }\end{array}$ & $\begin{aligned} 10 & (32) \\
9 & (29) \\
1 & (3)\end{aligned}$ & $\begin{array}{c}20(44) \\
19(42) \\
1(2)\end{array}$ & $\begin{array}{l}0.29 \\
0.24 \\
0.79\end{array}$ \\
\hline
\end{tabular}

Note: Data is presented as either no. (\%), mean \pm standard deviation, or median (interquartile range)

${ }^{*}$ Significance was determined using the unpaired $t$-test and chi-square test. ${ }^{\dagger} p<0.05$ (i.e. statistically significant). ${ }^{\ddagger} p<0.01$ (i.e. highly statistically significant). 8-OHdG: 8-hydroxy-2'-deoxyguanosine; ACE: angiotensin-converting enzyme; ACR: albumin/creatinine ratio (in urine); ARB: angiotensin receptor blocker; BMI: body mass index; eGFR: estimated glomerular filtration rate; HbA1c: haemoglobin A1c; HDL-C: high-density lipoprotein-cholesterol; HT: hypertension; MBP: mean blood pressure; PWV: pulse wave velocity; T2DM: type 2 diabetes mellitus.

significantly longer duration of DM $(p<0.01)$; higher levels of $\operatorname{MBP}(p<0.01)$, plasma glucose $(p=0.04), \operatorname{PWV}(p=0.02)$ and ACR $(p=0.04)$; a higher prevalence of retinopathy $(p=0.02)$; and a higher use of antidiabetic $(p=0.04)$ and antihypertensive agents (all types of agents except $\beta$-blockers) $(p<0.01$ ), when compared to T2DM patients without HT. However, there was no significant difference in the 8-OHdG levels of T2DM patients with and without HT.

In our cohort, T2DM patients with nephropathy showed higher levels of 8-OHdG (8.6 [6.4-10.9] ng/mg creatinine) and PWV $(1,549 \pm 207 \mathrm{~cm} / \mathrm{s})$ when compared to T2DM patients without nephropathy (8-OHdG 8.3 [5.9-10.4] ng/mg creatinine, $p=0.55$; PWV 1,527 $\pm 307 \mathrm{~cm} / \mathrm{s}, \mathrm{p}=0.75$ ), although the difference was not statistically significant. Similarly, the level of 8 -OHdG in T2DM patients with retinopathy
(7.9 [5.5-11.4] ng/mg creatinine) was not significantly different from that in T2DM patients without retinopathy (8.8 [6.5-10.2] ng/mg creatinine) $(p=0.62)$. However, T2DM patients with retinopathy showed a significantly higher level of PWV $(1,640 \pm 305 \mathrm{~cm} / \mathrm{s})$ than those without retinopathy $(1,485 \pm 253 \mathrm{~cm} / \mathrm{s})(\mathrm{p}=0.03)$. The $8-\mathrm{OHdG}$ levels of T2DM patients with and without HT were also compared to determine whether there was any difference between patients who were treated with antidiabetic, antihypertensive or lipid-lowering agents and those that were not. No significant differences were found in the 8-OHdG levels of these patients (data not shown).

Results of the correlation analysis of PWV with other variables, including 8-OHdG levels, are presented in Table II. Among the T2DM patients without HT, simple linear correlation analysis revealed that there was a significant positive correlation 
Table II. Correlation analysis of pulse wave velocity with other measured variables.

\begin{tabular}{|c|c|c|c|c|}
\hline \multirow[t]{2}{*}{ Variable } & \multicolumn{2}{|c|}{ T2DM patients without HT } & \multicolumn{2}{|c|}{ T2DM patients with HT } \\
\hline & $\mathbf{r}^{*}(p$-value $)$ & $\beta^{+}(p$-value $)$ & $\mathbf{r}^{*}(p$-value $)$ & $\beta^{+}(p$-value $)$ \\
\hline Age & $0.37(0.04)^{\S}$ & Not extracted & $0.44(<0.01)^{\pi}$ & Not extracted \\
\hline Male gender & $0.14(0.45)$ & Not extracted & $0.21(0.16)$ & $0.30(<0.01)^{\natural}$ \\
\hline Current smoking habit & $-0.17(0.36)$ & Not extracted & $0.05(0.77)$ & Not extracted \\
\hline Duration of $\mathrm{T}^{2} \mathrm{DM}^{\ddagger}$ & $0.32(0.08)$ & Not extracted & $0.36(0.02)^{\S}$ & $0.34(<0.01)^{9}$ \\
\hline BMI & $-0.32(0.09)$ & $-0.43(0.01)^{\S}$ & $-0.53(<0.01)^{\pi}$ & $-0.47(<0.01)^{\text {ฯ }}$ \\
\hline MBP & $0.50(<0.01)^{\pi}$ & $0.59(<0.01)^{\natural}$ & $0.39(0.01)^{\S}$ & $0.35(<0.01)^{\pi}$ \\
\hline Total cholesterol & $0.15(0.44)$ & Not extracted & $0.01(0.96)$ & Not extracted \\
\hline HDL-C & $-0.25(0.17)$ & Not extracted & $0.25(0.09)$ & Not extracted \\
\hline Triglycerides ${ }^{\ddagger}$ & $0.17(0.37)$ & Not extracted & $-0.30(0.05)$ & Not extracted \\
\hline Plasma glucose & $0.05(0.81)$ & Not entered & $0.08(0.62)$ & Not entered \\
\hline Creatinine & $0.18(0.34)$ & Not extracted & $0.11(0.48)$ & Not extracted \\
\hline 8-OHdG* & $-0.22(0.22)$ & Not extracted & $0.33(0.03)^{\S}$ & $0.23(0.02)^{\S}$ \\
\hline eGFR & $-0.24(0.20)$ & Not extracted & $-0.14(0.36)$ & $-0.23(0.03)^{\S}$ \\
\hline $\mathrm{ACR}^{*}$ & $0.09(0.63)$ & Not entered & $-0.06(0.69)$ & Not entered \\
\hline Nephropathy & $-0.02(0.93)$ & Not extracted & $-0.03(0.86)$ & Not extracted \\
\hline Retinopathy & $0.05(0.81)$ & Not extracted & $0.25(0.10)$ & Not extracted \\
\hline Antidiabetic agents & $0.36(0.04)^{\S}$ & Not extracted & $0.17(0.28)$ & Not extracted \\
\hline Antihypertensive agents & - & - & $-0.23(0.13)$ & Not extracted \\
\hline Lipid-lowering agents & $-0.14(0.44)$ & Not extracted & $-0.09(0.55)$ & Not extracted \\
\hline
\end{tabular}

* Simple linear correlation analysis was performed using Pearson's test. ${ }^{\dagger}$ Stepwise multiple linear regression analysis of pulse wave velocity with other parameters. ${ }^{\ddagger}$ Parameters had skewed distributions on correlation analysis and were therefore log transformed. ${ }^{\S} \mathrm{p}<0.05$ (i.e. statistically significant). ${ }^{9} p<0.01$ (i.e. highly statistically significant). 8-OHdG: 8-hydroxy-2'-deoxyguanosine; ACR: albumin/creatinine ratio (in urine); BMI: body mass index; eGFR: estimated glomerular filtration rate; HbA1c: haemoglobin A1c; HDL-C: high-density lipoprotein-cholesterol; HT: hypertension; MBP: mean blood pressure; PWV: pulse wave velocity; T2DM: type 2 diabetes mellitus

between PWV and age, MBP and the use of antidiabetic agents. Subsequent stepwise multiple linear regression analysis identified $\mathrm{BMI}$ (inversely) and MBP (positively) as variables that were independently and significantly correlated with PWV. On the other hand, among the T2DM patients with HT, simple linear correlation analysis revealed a significantly inverse correlation between PWV and BMI, and significantly positive correlations between PWV and age, duration of DM, MBP and 8-OHdG levels. Subsequent stepwise multiple linear regression analysis identified BMI (inversely), eGFR (inversely), male gender (positively), duration of DM (positively), MBP (positively) and 8-OHdG (positively) as variables that were independently and significantly correlated with PWV.

\section{DISCUSSION}

We found an independent, significant and positive correlation between the level of urinary 8-OHdG (an oxidative stress marker) and PWV (an arterial stiffness index) in patients with fairly well-controlled T2DM and HT, but not in T2DM patients without HT. Our data indicates that, under the hypertensive state, oxidative stress may be responsible for arterial stiffness even in T2DM patients with fair glycaemic control. Clinicians should therefore be aware that there might be a need for oxidative stress management in this patient population to prevent CVD. The addition of specific anti- hypertensive medications suggested to have antioxidative effects, ${ }^{(16)}$ as well as the inclusion of nonpharmaceutical antioxidant therapy (e.g. exercise, vitamin supplementation, and a diet rich in fruits and vegetables) ${ }^{(28-31)}$ may be included in the treatment regimen of these patients.

Several studies have investigated the association between PWV and oxidative stress-related markers. ${ }^{(32-35)}$ For instance, a positive correlation has been reported between 8-iso(epi)prostaglandin F2 $\alpha$ (an F2-isoprostane) and PWV, ${ }^{(32-34)}$ although the markers and patient populations in these earlier studies differed from those used in the present study. Similarly, in a study that evaluated serum $8-\mathrm{OHdG}$ levels in patients with chronic kidney disease, Dalfino et al reported a positive correlation between PWV and 8-OHdG levels. ${ }^{(35)}$ In the present study, although we did not correlate PWV with the same variables as those selected by earlier authors, ${ }^{(32-35)}$ our results were consistent with their inferences. In effect, our findings broaden the current understanding of $8-\mathrm{OHdG}$ and its correlation with PWV to a population of T2DM patients with HT.

We found that 8-OHdG levels were not clearly different between T2DM patients with HT and those without, unlike a previous study by Espinosa et al, which had reported higher 8-OHdG levels in patients with HT than in patients without $\mathrm{HT}$. ${ }^{(13)}$ The $8-\mathrm{OHdG}$ levels of our study cohort were not higher than those reported in healthy people by Kato et al. ${ }^{(36)}$ Also, the 


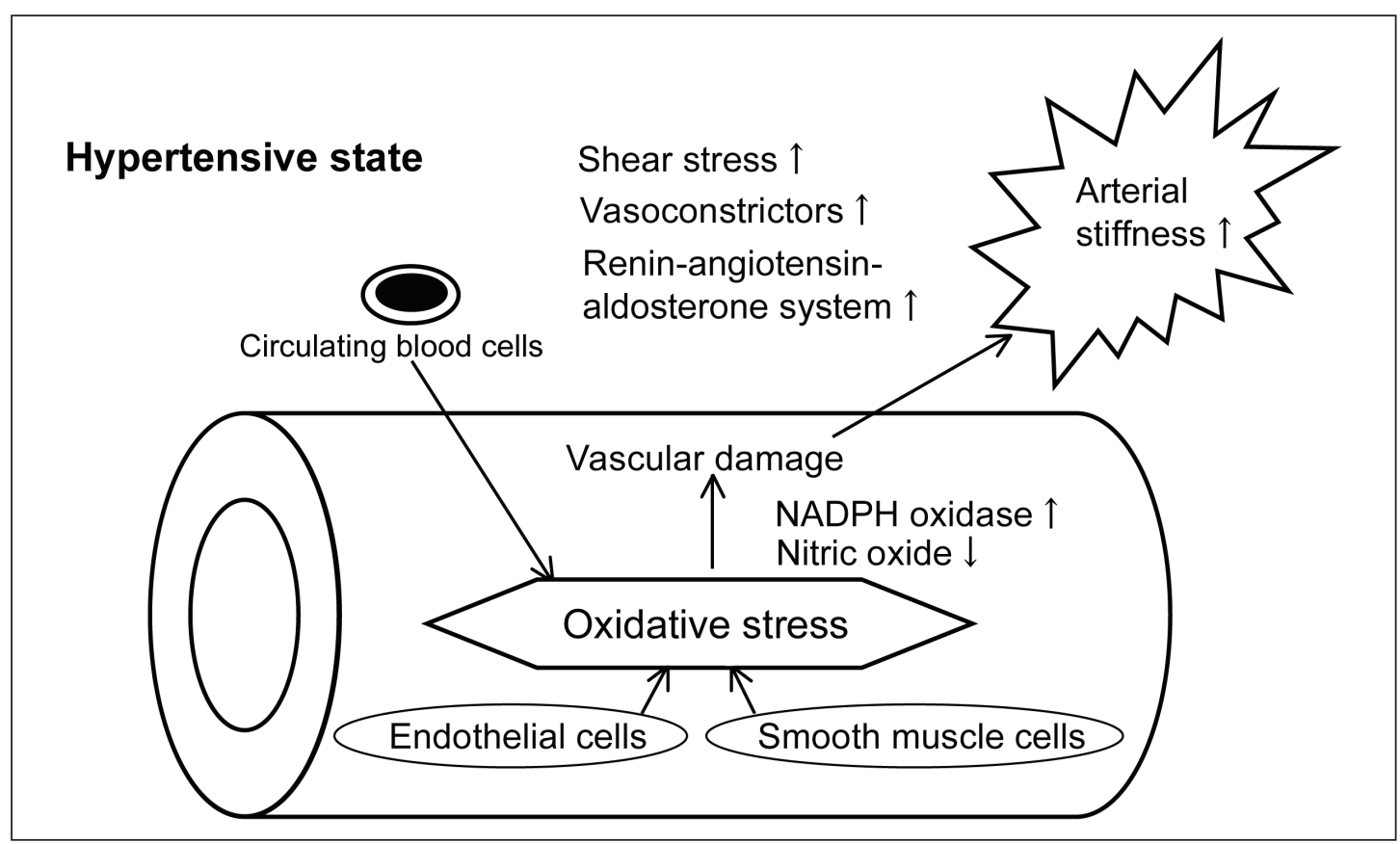

Fig. 1 Possible mechanism(s) accounting for the association between oxidative stress and arterial stiffness in hypertensive patients. NADPH: nicotinamide adenine dinucleotide phosphate

8-OHdG levels observed in our patients were similar to Miyashita et al's findings on T2DM patients with HT. (16) Nonetheless, further and more extensive studies will be necessary to determine the reason(s) behind the differences in 8-OHdG levels that have been observed by the many studies in the literature.

We found a significant correlation between 8-OHdG levels and PWV in T2DM patients with HT, indicating that the association between oxidative stress and arterial stiffness could be enhanced under the hypertensive state. Our results were partly in agreement with a recent study by Masugata et al, which found a positive correlation between $8-\mathrm{OHdG}$ and the cardio-ankle vascular index in patients with $\mathrm{HT}^{(19)}$ and with other earlier studies that reported that the role of $8-\mathrm{OHdG}$ could be amplified in the hypertensive state when compared to the diabetic state. ${ }^{(14,37)}$

Various possible biological mechanism(s) may be fundamental to the correlation between urinary $8-\mathrm{OHdG}$ levels and PWV in T2DM patients with HT (Fig. 1). The diabetic and/or hypertensive states are known oxidative stress conditions, ${ }^{(4,6-8)}$ and the association between oxidative stress and vascular damage is thought to be common to the pathophysiologies of HT and DM. ${ }^{(4-6)}$ Circulating blood cells (e.g. superoxideproducing cells, activated-T cells and platelets) and vascular cells (e.g. endothelial cells and smooth muscle cells) can produce oxidative stress. ${ }^{(14,38)}$ Studies have also reported that oxidative stress causes endothelial dysfunction, vascular inflammation, and arterial wall hypertrophy and fibrosis, thus resulting in increased arterial stiffness, with smooth muscle cell proliferation and increased synthesis of the extracellular matrix (i.e. elastin and collagen). ${ }^{(5,6,39)}$ Under the hypertensive state, the processes of vascular damage, which commence with the production of oxidative stress, proceed to the activation of nicotinamide adenine dinucleotide phosphate oxidase and the impaired bioavailability of nitric oxide, with shear stress also bringing into play factors such as the increased activity of vasoconstrictors (e.g. endothelin-1) and the activation of the renin-angiotensin-aldosterone system. ${ }^{(5,6,9,39,40)}$ Oxidative stress, together with factors involved in the hypertensive state, may therefore enhance arterial stiffness, thus accounting for the correlation of 8-OHdG levels with PWV in T2DM patients with HT.

We found that, in T2DM patients, male gender (in T2DM patients with $\mathrm{HT}$ ), duration of DM (in T2DM patients with $\mathrm{HT}$ ) and MBP (in T2DM patients with and without HT) were independently, significantly and positively correlated with PWV, while BMI (in T2DM patients with and without HT) and eGFR (in T2DM patients with HT) were independently, significantly and inversely correlated with PWV. The correlation between these variables and arterial stiffness, as indicated by our results, were also seen in previous studies - male gender, ${ }^{(41,42)}$ $\mathrm{BP}^{(43,44)}$ duration of $\mathrm{DM}^{(45)} \mathrm{eGFR}^{(46)}$ and $\mathrm{BMI}^{(47,48)}$ - although the characteristics of the various study populations were different. Although the inverse correlation between BMI and arterial stiffness in our study was unexpected, we found that similar results had also been reported previously. ${ }^{(47,48)}$

There were some limitations to the present study. The sample size was relatively small. Similarly, the inclusion of hypertensive patients without DM as control patients might have been better for comparison purposes. Our study had a cross-sectional design, and therefore could not entirely define the cause-and-effect relationship between 8-OHdG levels and PWV. While 8-OHdG levels are generally acknowledged to be an indicator of oxidative damage and atherogenesis, 
contradictory associations have also been reported in the literature, with a recent study by Huh et al suggesting that 8-OHdG might have an inhibitory role on atherosclerosis. ${ }^{(49)}$ Only patients with a specific level of glycaemic control were enrolled in our study. We found no significant differences between the 8-OHdG levels of T2DM patients with and without $\mathrm{HT}$, weak correlations between $8-\mathrm{OHdG}$ levels and the use of prescribed agents, and weak correlations between diabetic complications and PWV. However, the small sample size and the predetermined level of glycaemic control in our study might have partly accounted for our results. In contrast to our study, Aso et al demonstrated a significant association between PWV and diabetic complications. ${ }^{(50)}$ As various measures other than PWV measurement can be used for the evaluation of arterial stiffness, comparative studies that include the assessment of these variables may be useful for confirming our findings. ${ }^{(15,16,19)}$ The evaluation of markers associated with endothelial dysfunction would also be useful in this context. Therefore, future prospective studies, with larger and varied populations, that evaluate the many markers involved with oxidative stress are warranted.

In summary, we report an independent, significant and positive correlation between urinary $8-\mathrm{OHdG}$ levels and PWV in T2DM patients with HT, but not in those without HT. Our findings suggest that oxidative stress could be associated with the development of arterial stiffness in patients with fairly well controlled T2DM under the hypertensive state. Further studies will be necessary to clarify the biological mechanism(s) responsible, as well as the clinical relevance of the aforementioned relationship.

\section{REFERENCES}

1. Sheu WH, Rosman A, Mithal A, et al. Addressing the burden of type 2 diabetes and cardiovascular disease through the management of postprandial hyperglycaemia: an Asian-Pacific perspective and expert recommendations. Diabetes Res Clin Pract 2011; 92:312-21.

2. Hu FB. Globalization of diabetes: the role of diet, lifestyle, and genes. Diabetes Care 2011; 34:1249-57.

3. Chung N, Baek S, Chen MF, et al. Expert recommendations on the challenges of hypertension in Asia. Int J Clin Pract 2008; 62:1306-12.

4. Nishikawa T, Sasahara T, Kiritoshi S, et al. Evaluation of urinary 8-hydroxydeoxy-guanosine as a novel biomarker of macrovascular complications in type 2 diabetes. Diabetes Care 2003; 26:1507-12.

5. Stehouwer CD, Henry RM, Ferreira I. Arterial stiffness in diabetes and the metabolic syndrome: a pathway to cardiovascular disease. Diabetologia 2008; 51:527-39.

6. Montezano AC, Touyz RM. Oxidative stress, Noxs, and hypertension: experimental evidence and clinical controversies. Ann Med 2012; 44 Suppl 1:S2-16.

7. Piconi L, Quagliaro L, Ceriello A. Oxidative stress in diabetes. Clin Chem Lab Med 2003; 41:1144-9.

8. Wu LL, Chiou CC, Chang PY, Wu JT. Urinary 8-OHdG: a marker of oxidative stress to DNA and a risk factor for cancer, atherosclerosis and diabetics. Clin Chim Acta 2004; 339:1-9.

9. Negishi $H$, Njelekela $M$, Ikeda $K$, et al. Assessment of in vivo oxidative stress in hypertensive rats and hypertensive subjects in Tanzania, Africa. Hypertens Res 2000; 23:285-9.

10. Xu S, Touyz RM. Reactive oxygen species and vascular remodelling in hypertension: still alive. Can J Cardiol 2006; 22:947-51.

11. Leinonen J, Lehtimäki T, Toyokuni $S$, et al. New biomarker evidence of oxidative DNA damage in patients with non-insulin-dependent DM. FEBS Lett 1997; 417:150-2.

12. Kanauchi M, Nishioka $\mathrm{H}$, Hashimoto T. Oxidative DNA damage and tubulointerstitial injury in diabetic nephropathy. Nephron 2002; 91:327-9.

13. Espinosa O, Jiménez-Almazán J, Chaves FJ, et al. Urinary 8-oxo-7,8dihydro-2'-deoxyguanosine (8-oxo-dG), a reliable oxidative stress marker in hypertension. Free Radic Res 2007; 41:546-54.

14. Roselló-Lletí E, de Burgos FG, Morillas P, et al. Impact of cardiovascular risk factors and inflammatory status on urinary $8-\mathrm{OHdG}$ in essential hypertension. Am J Hypertens 2012; 25:236-42.

15. Nagayama D, Saiki A, Endo K, et al. Improvement of cardio-ankle vascular index by glimepiride in type 2 diabetic patients. Int J Clin Pract 2010; 64:1796-801.

16. Miyashita Y, Saiki A, Endo K, et al. Effects of olmesartan, an angiotensin II receptor blocker, and amlodipine, a calcium channel blocker, on CardioAnkle Vascular Index (CAVI) in type 2 diabetic patients with hypertension. J Atheroscler Thromb 2009; 16:621-6.

17. Li CH, Wu JS, Yang YC, et al. Increased arterial stiffness in subjects with impaired glucose tolerance and newly diagnosed diabetes but not isolated impaired fasting glucose. J Clin Endocrinol Metab 2012; 97:E658-62.

18. Barengo NC, Tuomilehto JO. Blood pressure treatment target in patients with diabetes mellitus-current evidence. Ann Med 2012; 44 Suppl 1:S36-42.

19. Masugata H, Senda S, Murao K, et al. Association between urinary 8-hydroxydeoxyguanosine, an indicator of oxidative stress, and the cardioankle vascular index in hypertensive patients. J Atheroscler Thromb 2012; 19:747-55.

20. Yamashina A, Tomiyama H, Takeda K, et al. Validity, reproducibility, and clinical significance of noninvasive brachial-ankle pulse wave velocity measurement. Hypertens Res 2002; 25:359-64.

21. Oomichi T, Emoto M, Tabata T, et al. Impact of glycemic control on survival of diabetic patients on chronic regular hemodialysis: a 7-year observational study. Diabetes Care 2006; 29:1496-500.

22. Chobanian AV, Bakris GL, Black HR, et al. Seventh report of the Joint National Committee on Prevention, Detection, Evaluation, and Treatment of High Blood Pressure. Hypertension 2003; 42:1206-52.

23. Imai E, Horio M, Nitta K, et al. Estimation of glomerular filtration rate by the MDRD study equation modified for Japanese patients with chronic kidney disease. Clin Exp Nephrol 2007; 11:41-50.

24. Toyokuni S, Tanaka T, Hattori Y, et al. Quantitative immunohistochemical determination of 8-hydroxy-2'-deoxyguanosine by a monoclonal antibody N45.1: its application to ferric nitrilotriacetate-induced renal carcinogenesis model. Lab Invest 1997; 76:365-74.

25. Yoshida R, Ogawa Y, Kasai H. Urinary 8-oxo-7,8-dihydro-2'-deoxyguanosine values measured by an ELISA correlated well with measurements by high-performance liquid chromatography with electrochemical detection. Cancer Epidemiol Biomarkers Prev 2002; 11:1076-81.

26. Rossi MC, Nicolucci A, Pellegrini F, et al. Identifying patients with type 2 diabetes at high risk of microalbuminuria: results of the DEMAND (Developing Education on Microalbuminuria for Awareness of reNal and cardiovascular risk in Diabetes) Study. Nephrol Dial Transplant 2008; 23:1278-84.

27. Wilkinson CP, Ferris FL 3rd, Klein RE, et al. Proposed international clinical diabetic retinopathy and diabetic macular edema disease severity scales. Ophthalmology 2003; 110:1677-82.

28. Thompson HJ, Heimendinger J, Haegele A, et al. Effect of increased vegetable and fruit consumption on markers of oxidative cellular damage. Carcinogenesis 1999; 20:2261-6.

29. Miquel J. Can antioxidant diet supplementation protect against age-related mitochondrial damage? Ann N Y Acad Sci 2002; 959:508-16.

30. Nojima H, Watanabe H, Yamane K, et al. Effect of aerobic exercise training on oxidative stress in patients with type 2 diabetes mellitus. Metabolism 2008; 57:170-6.

31. Ozkanlar S, Akcay F. Antioxidant vitamins in atherosclerosis - animal experiments and clinical studies. Adv Clin Exp Med 2012; 21:115-23.

32. Kals J, Kampus P, Kals M, et al. Impact of oxidative stress on arterial elasticity in patients with atherosclerosis. Am J Hypertens 2006; 19:902-8.

33. Kals J, Kampus $P$, Kals $M$, et al. Inflammation and oxidative stress are associated differently with endothelial function and arterial stiffness in healthy subjects and in patients with atherosclerosis. Scand J Clin Lab Invest 2008; 68:594-601. 
34. Ha CY, Kim JY, Paik JK, et al. The association of specific metabolites of lipid metabolism with markers of oxidative stress, inflammation and arterial stiffness in men with newly diagnosed type 2 Diabetes. Clin Endocrinol (Oxf) 2012; 76:674-82.

35. Dalfino G, Simone S, Porreca S, et al. Bone morphogenetic protein-2 may represent the molecular link between oxidative stress and vascular stiffness in chronic kidney disease. Atherosclerosis 2010; 211:418-23.

36. Kato M, lida M, Goto Y, Kondo T, Yajima I. Sunlight exposure-mediated DNA damage in young adults. Cancer Epidemiol Biomarkers Prev 2011; 20:1622-8.

37. Abdilla N, Tormo MC, Fabia MJ, et al. Impact of the components of metabolic syndrome on oxidative stress and enzymatic antioxidant activity in essential hypertension. J Hum Hypertens 2007; 21:68-75.

38. Martinet W, Knaapen MW, De Meyer GR, Herman AG, Kockx MM. Elevated levels of oxidative DNA damage and DNA repair enzymes in human atherosclerotic plaques. Circulation 2002; 106:927-32.

39. Mazzone T, Chait A, Plutzky J. Cardiovascular disease risk in type 2 diabetes mellitus: insights from mechanistic studies. Lancet 2008; 371:1800-9.

40. Ogawa S, Mori T, Nako K, Ito S. Combination therapy with renin-angiotensin system inhibitors and the calcium channel blocker azelnidipine decreases plasma inflammatory markers and urinary oxidative stress markers in patients with diabetic nephropathy. Hypertens Res 2008; 31:1147-55.

41. Nordstrand N, Gjevestad E, Dinh KN, et al. The relationship between various measures of obesity and arterial stiffness in morbidly obese patients. BMC Cardiovasc Disord 2011; 11:7.

42. Lim S, Choi HJ, Shin H, et al. Subclinical atherosclerosis in a communitybased elderly cohort: the Korean Longitudinal Study on Health and Aging.
Int J Cardiol 2012; 155:126-33.

43. Tomita H, Kawamoto R, Tabara Y, Miki T, Kohara K. Blood pressure is the main determinant of the reflection wave in patients with type 2 diabetes. Hypertens Res 2008; 31:493-9.

44. Xu L, Jiang CQ, Lam TH, et al. Brachial-ankle pulse wave velocity and cardiovascular risk factors in the non-diabetic and newly diagnosed diabetic Chinese: Guangzhou Biobank Cohort Study-CVD. Diabetes Metab Res Rev 2010; 26:133-9.

45. Kim WJ, Park CY, Park SE, et al. The association between regional arterial stiffness and diabetic retinopathy in type 2 diabetes. Atherosclerosis 2012; 225:237-41.

46. Nakagawa N, Takahashi F, Chinda J, et al. A newly estimated glomerular filtration rate is independently associated with arterial stiffness in Japanese patients. Hypertens Res 2008; 31:193-201.

47. Maple-Brown LJ, Piers LS, O'Rourke MF, Celermajer DS, O'Dea K. Central obesity is associated with reduced peripheral wave reflection in Indigenous Australians irrespective of diabetes status. J Hypertens 2005; 23:1403-7.

48. Budimir D, Jeroncic A, Gunjaca G, et al. Sex-specific association of anthropometric measures of body composition with arterial stiffness in a healthy population. Med Sci Monit 2012; 18:CR65-71.

49. Huh JY, Son DJ, Lee Y, et al. 8-Hydroxy-2-deoxyguanosine prevents plaque formation and inhibits vascular smooth muscle cell activation through Rac1 inactivation. Free Radic Biol Med 2012; 53:109-21.

50. Aso K, Miyata M, Kubo T, et al. Brachial-ankle pulse wave velocity is useful for evaluation of complications in type 2 diabetic patients. Hypertens Res 2003; 26:807-13. 